\title{
INSPECCIÓN DE AISLADORES EN LÍNEAS DE TRANSMISIÓN ELÉCTRICA USANDO INTELIGENCIA ARTIFICIAL
}

\section{POWER LINE INSULATORS INSPECTION BASED ON ARTIFICIAL INTELLIGENCE}

\author{
Ing. Sergio Luis Beleño Díaz, \\ PhD. Carol Viviana Martínez Luna**, \\ PhD. Iván Fernando Mondragón Bernal**, \\ PhD. Carlos Alberto Parra Rodríguez**. \\ "Universidad De Pamplona, Facultad de Ingenierías y Arquitectura, \\ Pamplona, Norte de Santander, Colombia. \\ E-mail: sergio.beleno@unipamplona.edu.co. \\ ${ }^{* *}$ Pontificia Universidad Javeriana, Sistemas Inteligentes, Robótica y Percepción. \\ Bogotá, Bogotá, Colombia. \\ E-mail: \{carolmartinez, imondragon, carlos.parra $\} @$ javeriana.edu.co.
}

Resumen: Uno de los procesos más importantes en la inspección de líneas de transmisión eléctrica es la detección de fallas en aisladores eléctricos. El defecto más común encontrado en los aisladores eléctricos es el quiebre de discos dentro de la cadena de aisladores. El uso de métodos tradicionales de segmentación por binarización indican una pobre capacidad para detectar un aislador si hay muchos cambios en el medio en el que se encuentra. Un algoritmo de inteligencia artificial conocido como You Only Look Once (YOLO) se usa para detectar y localizar los aisladores eléctricos a partir de imágenes de torres eléctricas de alta tensión. Posteriormente a la localización de los aisladores eléctricos, se realiza un escalado al doble del tamaño de la imagen original del aislador eléctrico usando un interpolador cúbico. De tal forma que le permita al supervisor de las líneas eléctricas de alta tensión realizar una correcta visualización de los aisladores a inspeccionar. La arquitectura de redes neuronales convolucionales MobileNet empleando el algoritmo YOLO, presentó resultados superiores en precisión y velocidad de ejecución con respecto a las arquitecturas Full YOLO e InceptionV3.

Palabras clave: Aisladores eléctricos, Redes neuronales artificiales, YOLO, Detección de objetos, Vehículos aéreos no tripulados.

\begin{abstract}
The failures detection on electrical insulators is a very important process in the task of powerline inspection. One of the usual mistakes found in this process is the breaking of disks in the chain of insulators. The use of classic techniques of segmentation in images has a weak ability to detect insulators if there are changes in the background. Instead, the artificial intelligent methods could be used to detect and locate the electrical insulators. In particular, You Only Look Once (YOLO) is proposed in this paper with this purpose. After locating the insulator, a process of scaling is performed using cubic interpolation to improve the image visualization to the human supervisor. The MobileNet convolutional neural network architecture in the YOLO algorithm, presents better results in precision and execution speed compared to the Full YOLO and InceptionV3 architectures.
\end{abstract}

Keywords: Electrical isolators, Artificial neural networks, YOLO, Object detection, Unmanned aerial vehicles. 


\section{INTRODUCCIÓN}

Uno de los procesos importantes en la inspección de líneas de transmisión eléctrica es la detección de fallas en aisladores eléctricos de alta tensión (J. Han et al., 2019; Li et al., 2017). Algoritmos de inteligencia artificial han realizado la detección de quiebres en aisladores con las imágenes tomadas por vehículos aéreos no tripulados, con resultados erróneos en algunas de sus detecciones, de manera que para certificar un proceso de inspección totalmente correcto en la detección de fallas en aisladores, es necesario lograr un porcentaje de precisión en la detección que logre o supere un 90\% de precisión sobre las imágenes de líneas de transmisión en el dataset de testeo (J. Han et al., 2019; Li et al., 2017).

En las pruebas realizadas por algoritmos para detectar los aisladores eléctricos en redes de alta tensión se encontraban errores de detección en cuanto a falsos positivos y falsos negativos ( $\mathrm{Li}$ et al., 2017). En caso de la detección de una falla del sistema donde el sistema de aisladores se encuentra en buen estado, es una detección que se conoce como falso positivo (Berk et al., 2017), como se muestra en la Figura 1.

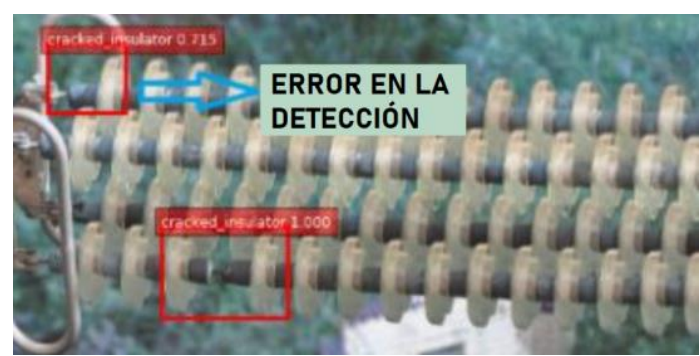

Fig. 1. Sistema de detección reconoce una falla erróneamente en un aislador de las líneas eléctricas de alta tensión. Fuente: (Li et al., 2017).

En el caso de una falta en la detección de una falla dentro del sistema de aisladores se presenta un falso negativo (Berk et al., 2017), como se muestra en la Figura 2.

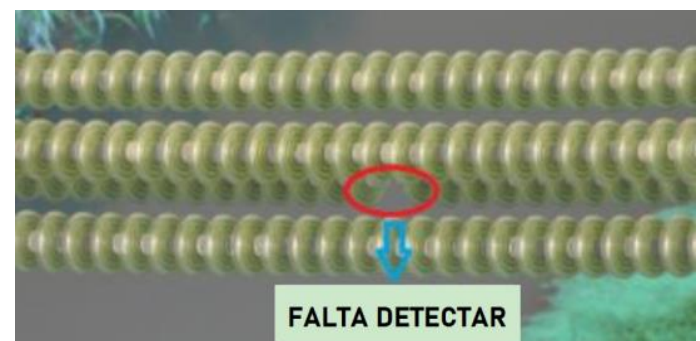

Fig. 2. Sistema de detección no reconoce la falla en uno de los aisladores de las líneas eléctricas de alta tensión. Fuente: (Li et al., 2017).
Metodos más recientes en la detección de fallas en aisladores eléctricos usan una binarización con respecto al color (J. Han et al., 2019). Por medio de la binarización se segmentan los aisladores eléctricos dentro de la imagen. Luego se le aplican filtros morfológicos para obtener una forma definida del aislador eléctrico, para luego ser procesado mediante redes neuronales convolucionales para detectar los defectos en el aislador como se muestra en la Figura 3.

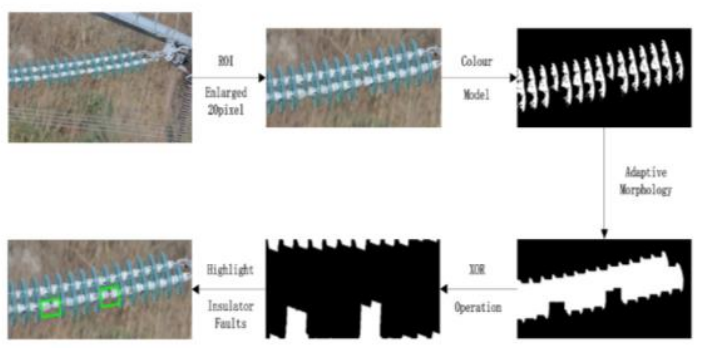

Fig. 3. Diagrama de flujo de la detección de fallas múltiples del aislador eléctrico.

Fuente: (J. Han et al., 2019).

Sistema que detecta usando segmentación por binarización en la primera etapa de preprocesamiento, resultando tener una precisión superior a un $90 \%$ evaluándose en las imágenes de testeo (J. Han et al., 2019). Pero, el hecho de usar una etapa de binarización para la segmentación representa un problema. Dado que la binarización resulta contraproducente si el fondo de la imagen cuenta con colores similares al color del objeto a detectar (Židek \& Hošovský, 2014). Si el aislador fuera de un matiz marrón en vez de azul, el detector fallaría en la detección desde la etapa inicial del algoritmo. El aislador eléctrico seria confundido con la tierra del fondo de la imagen como se muestra en la figura 4 .

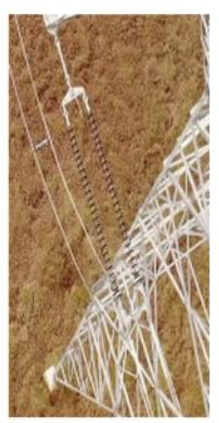

(a)

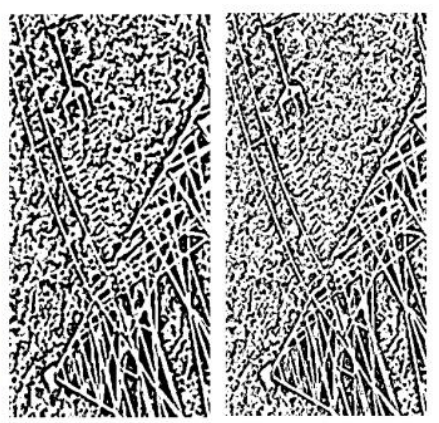

(b) (c)
Fig. 4. (a) Imagen original del aislador eléctrico, (b) Segmentación por binarización media adaptativa, (c) Segmentación por binarización adaptativa gaussiana. Fuente: Autores. 
La principal contribución de este artículo es el desarrollo de un algoritmo de inteligencia artificial usando la estructura del algoritmo You Only Look Once (YOLO) para detectar y localizar los aisladores en imágenes de líneas de transmisión eléctrica. Una vez realizada la localización de los aisladores eléctricos se realiza un escalado de la imagen al doble del tamaño original de la imagen del aislador usando un interpolador cúbico. El interpolador cúbico se caracteriza por desempeñar un redimensionamiento óptimo de las imágenes con respecto a interpoladores lineales e interpoladores de vecinos cercanos (D. Han, 2013). Este algoritmo le permite realizar al supervisor de las líneas de alta tensión una correcta visualización de los aisladores, facilitando el proceso de inspección sobre las líneas de transmisión eléctrica.

Este artículo este organizado de la siguiente manera: La sección 1, una introducción al tema de investigación. La sección 2, presenta el proceso de creación del conjunto de datos utilizado para el entrenamiento del sistema de inteligencia artificial. La sección 3, explica el método de inteligencia artificial a implementar. La sección 4, presenta los hiperparámetros usados para el entrenamiento del sistema de inteligencia artificial. La sección 5, presenta gráficas de desempeño en la implementación del algoritmo de inteligencia artificial. La sección 6, presenta los aportes relevantes del estudio realizado en este proyecto.

\section{DATASET}

En este artículo se presenta un banco de imágenes o Dataset que contiene 1100 imágenes etiquetadas de aisladores eléctricos. Se obtuvieron 600 imágenes etiquetadas a partir de la investigación en el área de las redes neuronales convolucionales por parte del dataset de Tao (Tao et al., 2020).

Sobre este se agregaron 500 imágenes de líneas de transmisión eléctrica tomadas a las afueras de la ciudad de Bogotá (Colombia), las cuales fueron etiquetadas usando el software Label-Img (Tzutalin, 2015), con el fin de realizar la detección de aisladores eléctricos en un estándar basado en la PASCAL VOC.

PASCAL VOC es un dataset conocido por ser usado en competencias con el desarrollo de algoritmos de detección y localización de objetos en imágenes (Mark et al., 2010). Un ejemplo del etiquetado de aisladores eléctricos bajo el estándar de la PASCAL VOC se presenta en la Figura 5, Figura 6 y Figura 7.

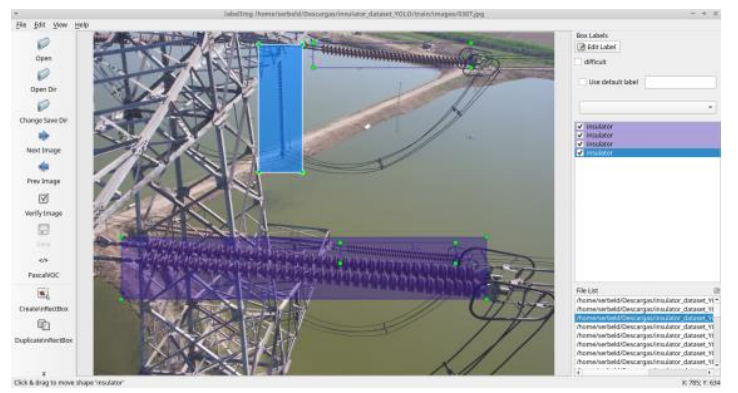

Fig. 5. Etiquetado de aisladores eléctricos en líneas de transmisión eléctrica usando el software Label-Img. Fuente: Autores

Para obtener una detección de los aisladores eléctricos en otros escenarios, se busca obtener imágenes de otras fuentes de información para hacer detecciones más robustas (Chan et al., 2018). Para tomar una segunda fuente de datos se realizaron vuelos con un vehículo aéreo no tripulado (Drone) con el fin de realizar la toma de datos de video en redes de transmisión eléctrica.

El drone fue operado manualmente sobre líneas eléctricas de $230 \mathrm{kV}$ energizadas en un tramo de más de $2 \mathrm{~km}$ a las afueras de la ciudad de Bogotá (Colombia). El trayecto del drone incluye cruce sobre ríos, zonas de bosque, praderas, cruces viales y montañas.

De los videos tomados se extrajeron 500 imágenes donde se visualizan aisladores eléctricos. De las imágenes obtenidas se etiquetaron la posición y nombre de etiqueta en de los aisladores eléctricos encontrados en cada imagen, como se muestra en la Figura 6.

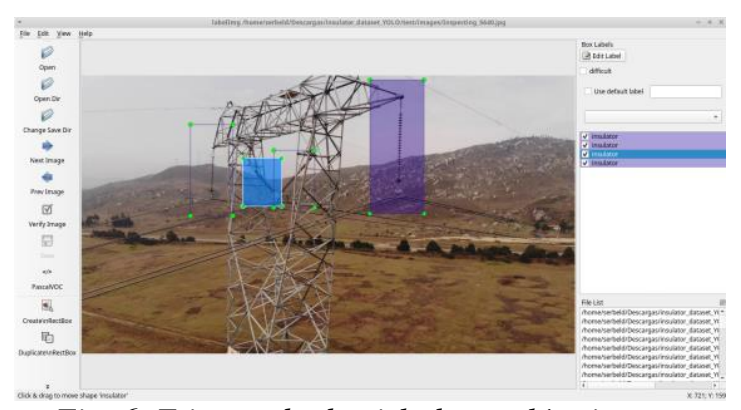

Fig. 6. Etiquetado de aisladores eléctricos en líneas de transmisión eléctrica usando el software Label-Img. Fuente: Autores

En dataset Tao se usó "insulator" para referirse a los aisladores eléctricos en las líneas de transmisión (Tao et al., 2020). Para mantener coincidencia en las etiquetas del dataset de Tao y las imágenes obtenidas, se etiquetaron las imágenes de la misma forma como se presenta en la Figura 7. 


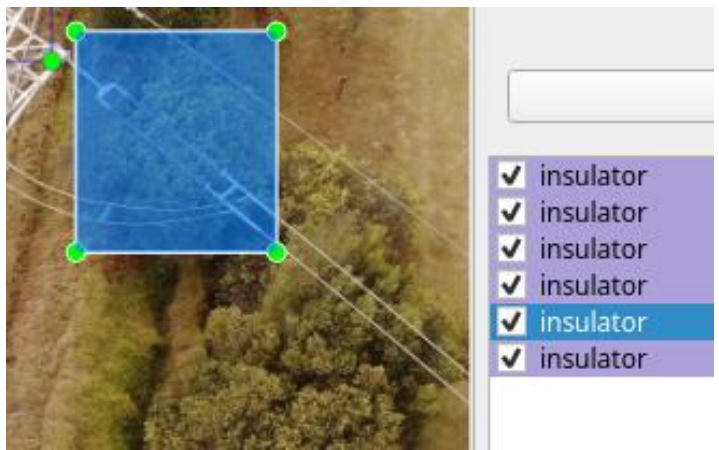

Fig. 7. Etiquetado de aisladores eléctricos en líneas de transmisión eléctrica usando el software Label-Img. Fuente: Autores

Al combinar ambos conjuntos de datos de Tao y los tomados por los autores, se obtienen en total 1100 imágenes etiquetadas. El Dataset obtenido se dividió en 3 Datasets para el entrenamiento, validación y testeo de las redes neuronales convolucionales.

El primer Dataset de datos es destinado a entrenamiento y contiene el $70 \%$ de todo el tamaño completo del Dataset. El segundo Dataset es destinado a validación y contiene el $15 \%$ de todo el tamaño completo del Dataset. El tercer Dataset es destinado a testeo y contiene el $15 \%$ faltante de todo el tamaño completo del Dataset,

\section{INTELIGENCIA ARTIFICIAL}

\subsection{Algoritmo You Only Look Once (YOLO)}

El algoritmo YOLO llamado así por sus siglas en inglés, es un sistema neuronal de detección multiclase usado en imágenes (Redmon et al., 2016). El algoritmo detecta las probabilidades de pertenencia a una clase e indica dónde se encuentra ubicado el objeto dentro de la imagen.

Al trabajar con inteligencia artificial existe cierta complejidad en cuanto al tiempo computacional para desarrollar detección de objetos en imágenes en tiempo real (Huang et al., 2019). Últimamente se han diseñado nuevas algoritmos que han demostrado gran desempeño en cuanto costo computacional, como lo es el sistema de inteligencia artificial You Only Look Once (YOLO) para la detección objetos en tiempo real (Huang et al., 2019).

El algoritmo YOLO genera los recuadros que se ajustan al mapa de probabilidades generado por las CNN como se muestra en la Figura 8 (Redmon et al., 2016; Redmon \& Farhadi, 2017).
Como último paso del algoritmo se obtienen las mejores aproximaciones de los recuadros que encierran a las clases con un parámetro de visión artificial llamado intersección sobre la unión o IOU por sus siglas en inglés. El IOU indica el grado de acoplamiento que existe entre los recuadros generados y los objetos detectados dentro del mapa de probabilidades de salida de la red neuronal convolucional del algoritmo YOLO (Rezatofighi et al., 2019). El principio de funcionamiento del algoritmo YOLO se muestra en la Figura 8.

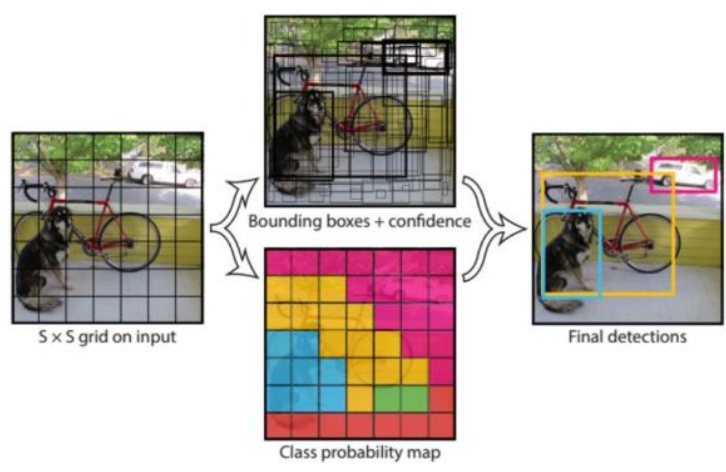

Fig. 8. Mapa de probabilidades del algoritmo YOLO. Fuente: (Redmon et al., 2016)

Para el entrenamiento del algoritmo YOLO es necesario ingresar tamaños predefinidos de lo recuadros que encierran los aisladores eléctricos dentro del Dataset propuesto (Redmon \& Farhadi, 2017). Para hallar el tamaño correcto para cada una de las cajas predefinidas, es usado el algoritmo K-means para hallar los tamaños base de recuadro más representativos dentro del Dataset.

\section{ALGORITMO DE DETECCIÓN DE AISLADORES ELÉCTRICOS}

El algoritmo propuesto para la detección de aisladores en el proceso de inspección de líneas eléctricas, se basa en el algoritmo YOLO para la detección de aisladores eléctricos en la extracción de imágenes para inspección de líneas de transmisión eléctrica.

Para el entrenamiento del algoritmo de inteligencia artificial se aplicaron diferentes tasas de aprendizaje y arquitecturas de redes neuronales convolucionales para garantizar el mejor desempeño del sistema (B.D.Hammel, 2019).

Se plantea el uso de la arquitectura Full YOLO por su excelente desempeño en la implementación del algoritmo YOLO con respecto a otros sistemas (Redmon et al., 2016; Redmon \& Farhadi, 2017). 
Se plantea el uso de la arquitectura Inception V3 por su balance de precisión y complejidad matemática con respecto a otras arquitecturas de redes neuronales convolucionales usando el dataset de 1.2 millones de imágenes conocido como ImageNet (Szegedy et al., 2016).

Inception V3 demostró un gran desempeño en la clasificación en el desafío ILSVRC (Russakovsky et al., 2014) en comparación con arquitecturas de redes convolucionales como GoogLeNet(Szegedy et al., 2015) y VGGNet (Simonyan \& Zisserman, 2015). (Pardo García A, Castellanos González L. 2017).

Se plantea el uso de la arquitectura MobileNet por su alta eficiencia y bajo costo computacional (Howard et al., 2017). La arquitectura MobileNet ha demostrado gran eficiencia en la detección de objetos, con una optimización tan buena que es recomendada para realizar aplicaciones en teléfonos inteligentes (Ignatov et al., 2019).

En todas las arquitecturas de redes neuronales convolucionales se usaron los mismos parámetros de entrenamiento en cada arquitectura. Se uso transfer learning para entrenar todas las arquitecturas a partir de los pesos de ImageNet (Sabatelli et al., 2019). Transfer learning es un método empleado para no tener que entrenar redes neuronales desde cero, permitiendo extraer unas características base para obtener mejores resultados en menor tiempo de entrenamiento de la red.

Los parámetros más significativos para el entrenamiento de las arquitecturas antes mencionadas son mostrados en la tabla 1.

Tabla 1: Parámetros usados en el entrenamiento del sistema de inteligencia artificial

\begin{tabular}{lc}
\hline \multicolumn{1}{c}{ Parámetros } & Valores \\
\hline Tamaño del lote por iteración & 4 \\
Número de épocas & 40 \\
Tamaños de recuadro predefinidos & 5 \\
Optimizador & Adam \\
Tamaño de entrada a la red & 416x416 pixeles \\
\hline
\end{tabular}

Con los parámetros mostrados en la tabla 1 se realizaron los entrenamientos del algoritmo YOLO usando las arquitecturas Full YOLO, InceptionV3 y MobileNet.

El algoritmo se entrena con el Dataset de entrenamiento, pero el algoritmo guarda su mejor modelo evaluando la arquitectura en cada época usando el Dataset de validación.

\section{RESULTADOS}

Se realizó el entrenamiento de las diferentes arquitecturas, donde se denota que el tiempo de entrenamiento por cada arquitectura fue entre 6 y 8 horas. En esta sección se presentan tablas de parámetros relevantes para medir el desempeño del sistema de inteligencia artificial. Estos resultados fueron obtenidos evaluando el sistema de inteligencia artificial en el Dataset de testeo.

En el proceso de entrenamiento de redes neuronales convolucionales la tasa de aprendizaje es el parámetro más relevante para buscar el mejor desempeño de una red (Wei, 2019). Para probar el desempeño del sistema de inteligencia artificial se usaron dos tasas de aprendizaje aplicadas sobre todas las arquitecturas de CNN planteadas.

Para medir la velocidad con la que ejecuta el algoritmo se utilizó la tasa de detecciones en fotogramas por unidad de segundo, también conocida como FPS por sus siglas en inglés (Frames Per Second).

En las tablas 2 y 3 donde muestra los resultados mas relevantes obtenidos del entrenamiento de algoritmo YOLO usando las arquitecturas Full YOLO, InceptionV3 y MobileNet.

\section{Tabla 2: Resultados de las arquitecturas de CNN usando una tasa de aprendizaje de 1e-03}

\begin{tabular}{cccc}
\hline Arquitectura & Precisión Media & IOU & FPS \\
\hline Full YOLO & $74.24 \%$ & $53.67 \%$ & 0.42 \\
InceptionV3 & NA & NA & NA \\
MobileNet & NA & NA & NA \\
\hline
\end{tabular}

Tabla 3: Resultados de las arquitecturas de CNN usando una tasa de aprendizaje de 1e-04

\begin{tabular}{cccc}
\hline Arquitectura & Precisión Media & IOU & FPS \\
\hline Full YOLO & $96.97 \%$ & $54.78 \%$ & 0.48 \\
InceptionV3 & $95.25 \%$ & $54.39 \%$ & 0.54 \\
MobileNet & $97.19 \%$ & $58.91 \%$ & 1.03 \\
\hline
\end{tabular}

El resultado de NA hace referencia a que no se consiguieron resultados concluyentes, debido a que las arquitecturas no detectaron ningún aislador eléctrico dentro de las imágenes encontradas en el Dataset de testeo.

En la tabla 3 se presenta que la arquitectura MobileNet logra superar al resto de las arquitecturas teniendo en cuenta la precisión media de detección del algoritmo, la intersección sobre la unión y la velocidad en la lectura de fotogramas por segundo. 


\subsection{Aplicación del algoritmo}

Se implemento la arquitectura MobileNet por su superioridad en la velocidad de lectura y alta precisión en la detección de aisladores eléctricos con respecto a las otras arquitecturas presentadas, esto comprobado por las pruebas realizadas con el Dataset de testeo de la tabla 3. Al implementar el algoritmo del sistema de detección se obtiene la ubicación de los aisladores eléctricos y su porcentaje de pertenencia a su clase, como se ve en la Figura 9.

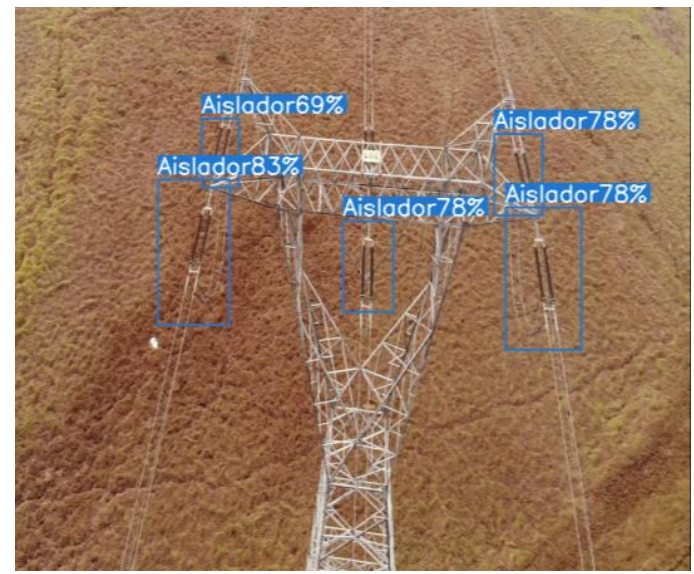

Fig. 9. Aplicación del algoritmo de detección de aisladores eléctricos. Fuente: Autores

Al tener la ubicación espacial de los aisladores se realiza un recorte con las dimensiones de cada uno de los aisladores. Al extraer las imágenes de los aisladores se realiza un proceso de escalado para aumentar el doble de su tamaño como se muestra en la Figura 10.
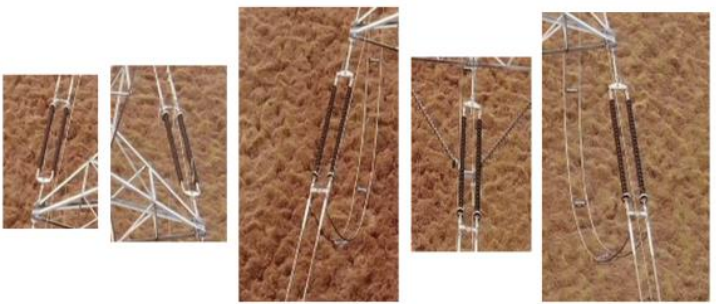

Fig. 10. Extracción de las imágenes de aisladores eléctricos usando el algoritmo de inteligencia artificial. Fuente: Autores

Es necesario un escalado para una mejor visualización de las imágenes de los aisladores, por lo que se implementó una interpolación cubica por su desempeño óptimo en cuanto al redimensionamiento de imágenes, en comparación con interpoladores lineales e interpoladores de vecinos cercanos, permitiendo obtener cada imagen lo menos pixelada posible con respecto al ojo humano (D. Han, 2013). Debido a sus características el interpolador cubico es ampliamente utilizado en el campo de software destinados a la edición de imagen y video.

Una demostración del algoritmo de inteligencia artificial en funcionamiento está disponible en el siguiente enlace: https://youtu.be/SfpS2-1VPXc

\subsection{Gráficas de desempeño ROC}

La curva característica de funcionamiento del receptor (ROC), es el gráfico que representa el comportamiento de la sensibilidad en contra de la especificidad de un sistema (Kumar \& Indrayan, 2011). Este es un método efectivo que tiene como fin evaluar el rendimiento de una prueba de diagnóstico o detección.

La curva de ROC se puede resumir en un solo valor normalizado conocido como el área bajo la curva o AUC (Muschelli, 2019), como se muestra en la Figura 11.

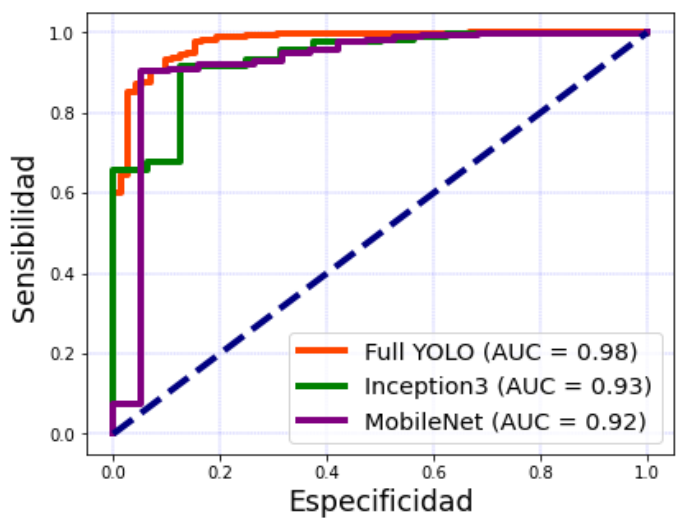

Fig. 11. Curva de sensibilidad vs especificidad usando una tasa de aprendizaje de 1e-04.

Fuente: Autores

La curva de sensibilidad vs especificidad muestra el crecimiento en la detección de falsos positivos con respecto a los verdaderos positivos en una escala normalizada mostrando un mejor desempeño en la arquitectura Full YOLO tomando en cuenta el parámetro AUC.

Aunque el desempeño de la arquitectura Full YOLO muestre un resultado creciente conforme a las detecciones realizadas en la Figura 11, en cuanto a valores numéricos en su precisión presenta un comportamiento diferente. Este comportamiento de mejor calidad para el análisis se afirma en la tabla 3 , se muestra una alta 
precisión en la detección de aisladores con la arquitectura MobileNet y se concreta con la gráfica 12, donde se muestra el desempeño de cada una de las arquitecturas empleadas con respecto a su precisión evaluadas en el Dataset de testeo.

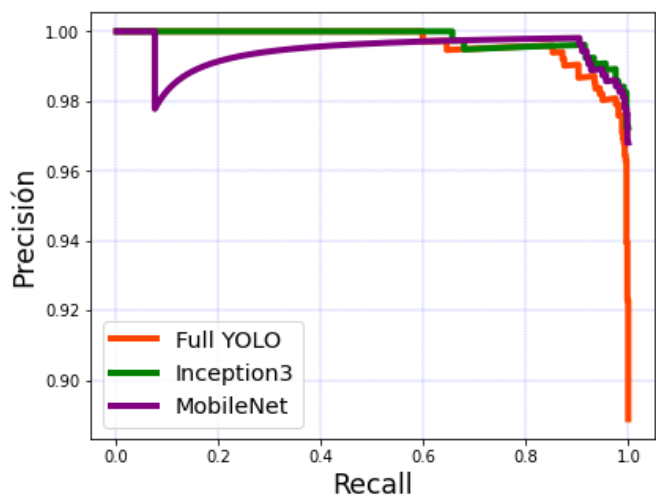

Fig. 12. Curva de precisión vs recall usando una tasa de aprendizaje de 1e-04. Fuente: Autores

De la Figura 12 se aprecia la curva de precisión vs recall donde se presenta la caída en la precisión en la arquitectura Full YOLO con respecto al recall en comparación con las arquitecturas InceptionV3 y MobileNet.

\section{CONCLUSIONES}

Se realizaron 3 Datasets usando el software de código abierto Label-Img, los Datasets creados se componen por entrenamiento, validación y testeo. Estos 3 Datasets fueron creados a partir de imágenes tomadas de líneas de transmisión eléctrica haciendo uso de drones, los Datasets propuestos en conjunto permiten realizar el entrenamiento del algoritmo YOLO para la detección y localización de aisladores eléctricos en imágenes usando un estándar de etiquetado de la PASCAL VOC.

El algoritmo YOLO se aplicó en videos y en imágenes del Dataset de testeo, logrando una precisión superior al $95 \%$ en la detección de aisladores eléctricos y una velocidad de procesamiento de 1 fotograma por segundo usando la arquitectura MobileNet. La arquitectura MobileNet empleada en el algoritmo YOLO, demostró ser la mejor arquitectura para la detección de aisladores eléctricos por su velocidad de respuesta y alta precisión porcentual. La arquitectura MobileNet superó a las arquitecturas InceptionV3 y Full YOLO, doblegando los resultados con respecto a la cantidad de fotogramas por segundo que es capaz de procesar el algoritmo.
Se extrajeron imágenes de aisladores eléctricos usando un algoritmo de inteligencia artificial permitiendo una visualización cercana de los elementos eléctricos contenidos en imágenes de líneas de transmisión eléctrica. La extracción de las imágenes de los aisladores eléctricos permite al supervisor realizar un fácil proceso de inspección de las líneas de transmisión eléctrica.

Métodos de interpolación para aumentar el tamaño de una imagen se ven pixelados por la pérdida de la calidad de la imagen al ser redimensionada (Dong et al., 2016). Algoritmos de inteligencia artificial están siendo recientemente investigados para obtener imágenes con super resolución, lo que implicaría una mejor visualización en los elementos de una imagen. Uno de los objetivos a futuro seria implementar algoritmos de inteligencia artificial para lograr obtener imágenes con super resolución para una visualización con mejores características de los elementos que componen las líneas de transmisión eléctricas.

\section{REFERENCIAS}

B.D.Hammel. (2019). What learning rate should I use? B.D.Hammel. http://www.bdhammel.com/learning-rates/

Berk, R., Heidari, H., Jabbari, S., Kearns, M., \& Roth, A. (2017). Fairness in Criminal Justice Risk Assessments: The State of the Art. Sociological Methods and Research, 1-43. https://doi.org/10.1177/0049124118782533

Chan, L., McGarey, P., \& Sclafani, J. A. (2018). Chapter 20 - Using Large Data Sets for Population-Based Health Research (J. I. Gallin, F. P. Ognibene, \& L. L. B. T.-P. and P. of C. R. (Fourth E. Johnson (eds.); pp. 293-302). Academic Press. https://doi.org/https://doi.org/10.1016/B9780-12-849905-4.00020-4

Dong, C., Loy, C. C., He, K., \& Tang, X. (2016). Image Super-Resolution Using Deep Convolutional Networks. IEEE Transactions on Pattern Analysis and Machine Intelligence, 38(2), 295-307.

Han, D. (2013). Comparison of Commonly Used Image Interpolation Methods. Iccsee. https://doi.org/10.2991/iccsee.2013.391

Han, J., Yang, Z., Zhang, Q., Chen, C., Li, H., Lai, S., Hu, G., Xu, C., Xu, H., Wang, D., \& Chen, R. (2019). A method of insulator faults detection in aerial images for high-voltage transmission lines inspection. Applied Sciences (Switzerland), 9(10), 1-22. https://doi.org/10.3390/app9102009 
Howard, A. G., Zhu, M., Chen, B., Kalenichenko, D., Wang, W., Weyand, T., Andreetto, M., \& Adam, H. (2017). MobileNets: Efficient Convolutional Neural Networks for Mobile Vision Applications. http://arxiv.org/abs/1704.04861

Huang, R., Pedoeem, J., \& Chen, C. (2019). YOLO-LITE: A Real-Time Object Detection Algorithm Optimized for Non-GPU Computers. Proceedings - 2018 IEEE International Conference on Big Data, Big Data 2018, 2503-2510.

Ignatov, A., Timofte, R., Chou, W., Wang, K., Wu, M., Hartley, T., \& Van Gool, L. (2019). AI Benchmark: Running deep neural networks on android smartphones. Lecture Notes in Computer Science (Including Subseries Lecture Notes in Artificial Intelligence and Lecture Notes in Bioinformatics), 11133 LNCS, 288-314. https://doi.org/10.1007/9783-030-11021-5 19

Kumar, R., \& Indrayan, A. (2011). Receiver operating characteristic (ROC) curve for medical researchers. Indian Pediatrics, 48(4), 277-287.

Li, S., Zhou, H., Wang, G., Zhu, X., Kong, L., \& Hu, Z. (2017). Cracked Insulator Detection Based on R-FCN. Journal of Physics: Conference Series, 1069(1). https://doi.org/10.1088/17426596/1069/1/012147

Mark, E., Luc, V.-G., I, W. C. K., John., W., \& Andrew, Z. (2010). The Pascal Visual Object Classes (VOC) Challenge. International Journal of Computer Vision, 88(2), 303-338.

Muschelli, J. (2019). ROC and AUC with a Binary Predictor: a Potentially Misleading Metric. Journal of Classification, 2020, 1-20. https://doi.org/10.1007/s00357-019-09345-1

Redmon, J., Divvala, S., Girshick, R., \& Farhadi, A. (2016). You only look once: Unified, realtime object detection. Proceedings of the IEEE Computer Society Conference on Computer Vision and Pattern Recognition, 2016-Decem, 779-788. https://doi.org/10.1109/CVPR.2016.91

Pardo García A, Castellanos González L. (2017). Automatización de Ambientes en Invernaderos Simulando Escenarios Futuros, Revista Colombiana de Tecnologías de Avanzada, ISSN: 1692-7257. Volumen1Número 29-2017.

Rezatofighi, H., Tsoi, N., Gwak, J., Sadeghian, A., Reid, I., \& Savarese, S. (2019). Generalized intersection over union: A metric and a loss for bounding box regression. Proceedings of the IEEE Computer Society Conference on Computer Vision and Pattern Recognition, 2019-June, 658-666.

https://doi.org/10.1109/CVPR.2019.00075

Russakovsky, O., Deng, J., Su, H., Krause, J., Satheesh, S., Ma, S., Huang, Z., Karpathy, A., Khosla, A., \& Bernstein, M. (2014). Imagenet large scale visual recognition challenge. Int. J. Comput. Vis., 1-42.

Sabatelli, M., Kestemont, M., Daelemans, W., \& Geurts, P. (2019). Deep transfer learning for art classification problems. Lecture Notes in Computer Science (Including Subseries Lecture Notes in Artificial Intelligence and Lecture Notes in Bioinformatics), 11130 LNCS(September), 631-646. https://doi.org/10.1007/978-3-030-110123_48

Simonyan, K., \& Zisserman, A. (2015). Very deep convolutional networks for large-scale image recognition. 3rd International Conference on Learning Representations, ICLR 2015 Conference Track Proceedings, 1-14.

Szegedy, C., Liu, W., Jia, Y., Sermanet, P., Reed, S., Anguelov, D., Erhan, D., Vanhoucke, V., \& Rabinovich, A. (2015). Going deeper with convolutions. Proceedings of the IEEE Computer Society Conference on Computer Vision and Pattern Recognition, 07-12-June. https://doi.org/10.1109/CVPR.2015.7298594

Szegedy, C., Vanhoucke, V., Ioffe, S., Shlens, J., \& Wojna, Z. (2016). Rethinking the Inception Architecture for Computer Vision. Proceedings of the IEEE Computer Society Conference on Computer Vision and Pattern Recognition, 2016-Decem, 2818-2826. https://doi.org/10.1109/CVPR.2016.308

Tao, X., Zhang, D., Wang, Z., Liu, X., Zhang, H., \& Xu, D. (2020). Detection of power line insulator defects using aerial images analyzed with convolutional neural networks. IEEE Transactions on Systems, Man, and Cybernetics: Systems, 50(4), 1486-1498. https://doi.org/10.1109/TSMC.2018.2871750

Tzutalin. (2015). LabelImg. Git Code LabelImg. ttps://github.com/tzutalin/labelImg

Wei, J. (2019). Forget the learning rate, decay loss. International Journal of Machine Learning and Computing, 9(3), 267-272. https://doi.org/10.18178/ijmlc.2019.9.3.797

Židek, K., \& Hošovský, A. (2014). Image thresholding and contour detection with dynamic background selection for inspection tasks in machine vision. International Journal of Circuits, Systems and Signal Processing, 8, 545-554. 